\title{
ARTí́CULO
}

\section{Composición de especies en la pesquería artesanal de enmalle de merluza común Merluccius gayi gayi en Chile central}

\author{
Species composition in the artisanal gillnet fishery of Chilean hake \\ Merluccius gayi gayi in central Chile
Dante Queirolo $^{1}$, José Merino ${ }^{1}$, Mauricio Ahumada ${ }^{1}$, Ivonne Montenegro', Erick Gaete ${ }^{1}$ y Roberto Escobar ${ }^{1}$

\begin{abstract}
${ }^{1}$ Escuela de Ciencias del Mar, Facultad de Recursos Naturales, Pontificia Universidad Católica de Valparaíso, Casilla 1020, Valparaíso, Chile. dante.queirolo@ucv.cl
\end{abstract}

\begin{abstract}
The species composition in commercial fisheries is important information to establish management measures that contributed to reduce the impact and ensure the sustainability of the activity. A study was conducted to identify and quantify the species caught in the artisanal gillnet fishery of Chilean hake (Merluccius gayi gayi) between January and September of 2010. The capture was registered in 98 fishing sets performed by 45 fishing vessels of 14 artisanal ports distributed between the Valparaíso and Biobío regions. The target specie was the most important species caught in all regions. The presence of 24 taxa as by-catch was registered nevertheless only 4 species had a relative frequency of occurrence higher than $50 \%$ of sets performed in some of the regions. These species were lemon crab (Cancer porteri), armed box crab (Platymera gaudichaudii), red squat lobster (Pleuroncodes monodon) and big eye flounder (Hippoglossina macrops), which accounted the highest proportion $(>70 \%)$ of total by-catch in number. Through classification and ordination methods based on species composition, 4 groups related to geographical regions and bathymetrical range were determined, while the mesh sizes used in the gillnets would have a minor influence on the explanation of the identified groups.
\end{abstract}

Key words: Gillnet, by-catch, Chilean hake, artisanal fishery, Chile

Resumen.- Se realizó un estudio para identificar y cuantificar las especies presentes en la pesquería artesanal de enmalle dirigida a merluza común (Merluccius gayi gayi) entre enero y septiembre de 2010. Se registró la captura en 98 lances de pesca realizados por 45 embarcaciones de 14 caletas distribuidas entre las regiones de Valparaíso y del Biobío. La especie objetivo fue la más relevante en las capturas en todas las regiones. La presencia de 24 taxones fueron registradas como fauna acompañante, no obstante sólo 4 especies presentaron una frecuencia relativa de ocurrencia mayor al $50 \%$ de los lances realizados en alguna de las regiones. Estas especies fueron jaiba limón (Cancer porteri), jaiba paco (Platymera gaudichaudii), langostino colorado (Pleuroncodes monodon) y lenguado (Hippoglossina macrops), las cuales explicaron la mayor proporción $(>70 \%$ ) de la fauna acompañante total en número. Mediante métodos de clasificación y ordenación basados en la composición de especies, se determinó la existencia de 4 grupos asociados a las regiones geográficas y al rango de profundidad donde se realizaron los lances de pesca, mientras que los tamaños de malla usados en las redes tendrían una influencia menor en la explicación de los grupos identificados.

Palabras clave: Enmalle, fauna acompañante, merluza común, pesca artesanal, Chile

\section{INTRODUCCIÓN}

La merluza común Merluccius gayi gayi Guichenot, 1848 se distribuye frente a la costa de Chile entre $23^{\circ}$ y $47^{\circ} \mathrm{S}$ en profundidades entre 50 y $500 \mathrm{~m}$ (Aguayo 1995). Esta especie constituye el principal recurso demersal de Chile central y sustenta una pesquería en la que participa una flota industrial y otra artesanal. Aun cuando se han establecido diversas medidas para el manejo de la pesquería (i.e., cierre de acceso, cuotas, veda biológica, etc.), la población de merluza común se ha reducido drásticamente en la última década, debido tanto a factores naturales (i.e., predación, canibalismo) como a la mortalidad por pesca ejercida por ambas flotas.

En el sector artesanal se registró un paulatino descenso en los rendimientos de pesca entre 2001 y 2006 
(SUBPESCA 2012) ${ }^{1}$ el cual motivó el reemplazo del espinel horizontal de fondo por la red de enmalle como principal aparejo utilizado en la pesquería. Este cambio se produjo gradualmente a partir de 2004, llegando en 2010 a ser el principal método de captura de merluza común por parte de la flota artesanal entre la Región de Valparaíso (V Región) y la Región del Biobío (VIII Región) (Queirolo et al. 2011). Al respecto, las redes de enmalle presentan algunas diferencias en su construcción entre localidades, siendo la más importante aquella relacionada con el tamaño de malla. Queirolo et al. (2011) señalan que el tamaño de malla predominante en las redes de la pesquería aumenta de norte a sur, específicamente de $50 \mathrm{~mm}$ a $83 \mathrm{~mm}$ desde la región de Valparaíso a la región del Biobío.

La selectividad, a la talla como entre especies, constituye información valiosa para establecer medidas de manejo en las pesquerías (Sparre \& Venema 1997). En este contexto, en la pesquería artesanal de merluza común se ha avanzado en la selectividad a la talla, habiéndose estimado curvas de selectividad en función a distintos tamaños de malla en trabajos recientes (Queirolo et al. 2013a, b). Así por ejemplo, las longitudes modales estimadas para ambos sexos combinados corresponden a 30,9, 40,2 y 43,9 cm de longitud total para tamaños de malla de 52, 68 y $76 \mathrm{~mm}$, respectivamente (Queirolo et al. 2013a). No obstante, persiste la falta de información respecto de la composición de especies y su participación relativa en las capturas, principalmente debido a la inexistencia de programas de observación a bordo en la operación de esta flota.

Para incrementar el conocimiento respecto a la interacción de los artes de pesca sobre especies noobjetivo y proporcionar una línea base para futuros estudios, se llevó a cabo el presente trabajo cuyo objetivo fue la identificación y cuantificación de especies que componen la captura con redes de enmalle en las principales áreas de operación de la flota merlucera artesanal.

\section{MATERIALES Y MÉTODOS}

El presente estudio fue realizado mediante el monitoreo de embarcaciones artesanales que operaron en pesca comercial frente a la costa centro-sur de Chile, específicamente entre $32^{\circ} 55^{\prime}$ y $38^{\circ} 20^{\prime}$ 'S, entre enero y septiembre de 2010. Dada la extensión del área, el esfuerzo de muestreo fue distribuido en 14 puertos base o caletas en los que se concentra el mayor desembarque artesanal de merluza común (Fig. 1). En total, se registró la captura por especie en 98 lances de pesca con redes de enmalle, los cuales fueron llevados a cabo por 45 embarcaciones. Cada embarcación usó el régimen operacional tradicional de cada zona, por lo cual entre las regiones V y VII (Región del Maule) el tiempo de reposo fluctuó entre 0,5 y $3 \mathrm{~h}$ (1,6 h en promedio), mientras que en la VIII Región fluctuó entre 21 y $24 \mathrm{~h}$ (22,3 h en promedio).

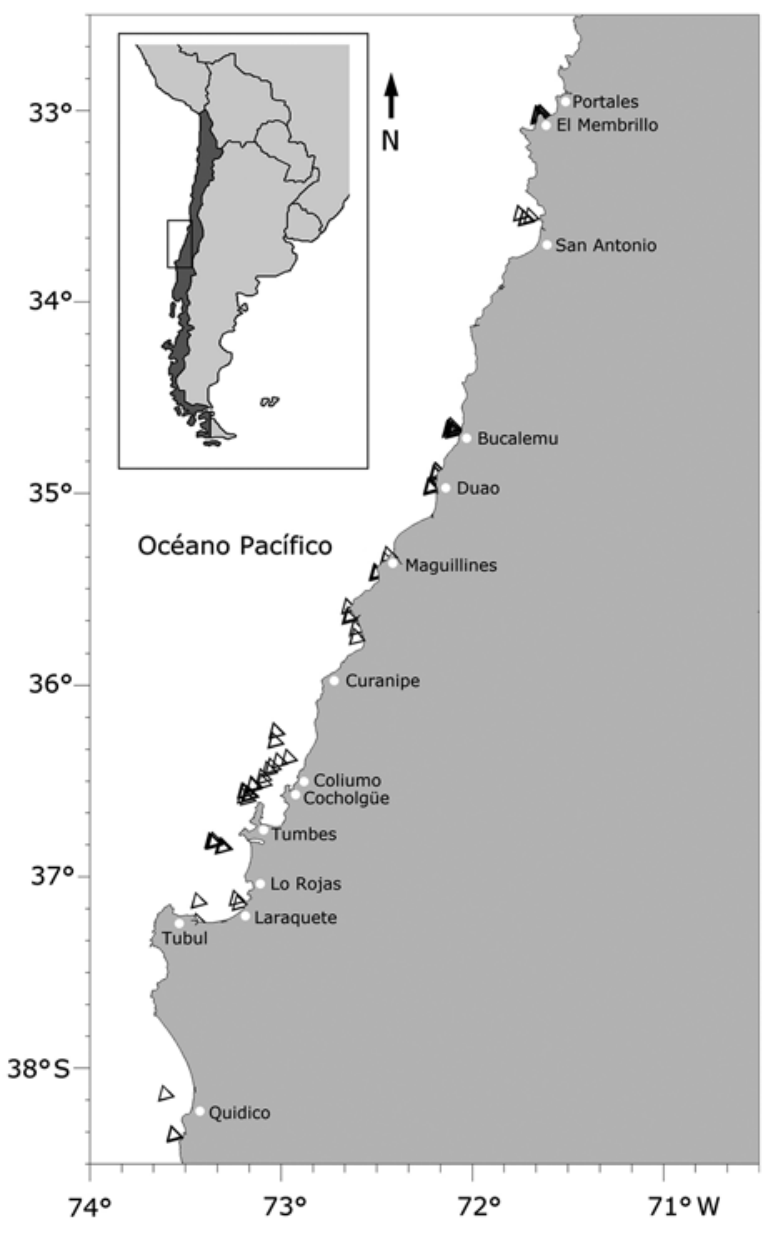

Figura 1. Distribución de las caletas y de los lances de pesca en el área de estudio / Distribution of the fishing coves and fishing sets in the study area

${ }^{1}$ SUBPESCA. 2012. Cuota global anual de captura de merluza común (Merluccius gayi gayi), año 2013. Informe Técnico (R. Pesq.) 215/2012: 1-43. Subsecretaría de Pesca, Valparaíso. 
Las redes de enmalle fueron las mismas que utilizan los pescadores artesanales en sus faenas habituales, construidas con paños de poliamida monofilamento de color verde, con variaciones en el tamaño de malla y diámetro del hilo, fluctuando entre 52 y $85 \mathrm{~mm}$ y entre 0,25 y $0,5 \mathrm{~mm}$, respectivamente. La longitud de las redes de enmalle fluctuó entre 420 y $1.510 \mathrm{~m}$, mientras que el embande (relación entre la longitud de la relinga superior y la longitud de la tela estirada) varió entre 0,4 y 0,55.

En cada lance se registró la posición de calado, tiempo de reposo, cantidad de paños calados, y captura por especie. La profundidad del fondo fue registrada como valor aproximado a partir de la información proporcionada por los pescadores. Todos los ejemplares capturados, tanto del recurso objetivo como de la fauna acompañante, fueron contabilizados en la embarcación y pesados en tierra. En algunos lances de alta abundancia, la captura total se estimó a partir del número de ejemplares en una muestra representativa ( $20 \%$ del total). La identificación de las especies se realizó a bordo con la ayuda de una guía de campo elaborada a partir de los trabajos de Meléndez \& Meneses (1989), Retamal (1981), Kong \& Meléndez (1991) y Pequeño (1997).

Se determinó la captura total en número y peso así como el rendimiento de captura por cada kilómetro de red por zona de pesca y región. Cada especie fue clasificada según su frecuencia relativa de ocurrencia (fro= frecuencia de ocurrencia/número de lances) en 4 categorías conforme a lo descrito por Santos et al. (2002): i) R= especies raras (So $<0,25)$, ii) $\mathrm{U}=$ especies poco comunes $(0,25 \leq \mathrm{So}<$ $0,50)$, iii) $\mathrm{C}=$ especies comunes $(0,50 \leq \mathrm{So}<0,75)$ y iv) $\mathrm{V}=$ especies muy comunes (So $\geq 0,75$ ).

Se realizó un análisis multivariado de la composición de las capturas según lo descrito por Stergiou et al. (2002, 2006). Se utilizó el análisis de agrupamiento (medias grupales) empleando el índice de similitud de Bray-Curtis (Field et al. 1982), el cual fue aplicado a los datos de captura estandarizada por lance (por $1 \mathrm{~km}$ lineal de red calada). Los factores asociados a cada lance de pesca correspondieron a la región (V, VI, VII y VIII), el tamaño de malla (52, 65, 73 y $85 \mathrm{~mm}$ ) y la profundidad $(<50,51-$ $100,101-150$ y $>151 \mathrm{~m})$.

Dada la participación de especies raras y especies muy comunes, los datos originales de captura estandarizada fueron transformados aplicando raíz cuarta según lo propuesto por Clarke \& Warwick (2001). También se realizó el análisis de ordenación mediante escalamiento multidimensional (MDS) con la misma información utilizada en el análisis de agrupamiento. Para verificar la adecuada representación en 2 dimensiones, se utilizó a modo de contraste el coeficiente de estrés, donde valores menores a 0,1 implican una buena representación de los datos y valores mayores a 0,2 una ordenación menos confiable (Clarke \& Warwick 2001). Las especies características que contribuyeron a la similitud de cada grupo fueron determinadas mediante el análisis de similitud porcentual (SIMPER).

\section{Resultados}

La captura total en 98 lances y $83.829 \mathrm{~m}$ de redes caladas fue $26.008 \mathrm{~kg}$ y 122.336 ejemplares (Tabla 1), identificándose 25 taxones de las cuales 13 fueron vertebrados y 12 invertebrados (Tabla 2). La merluza común, especie objetivo de la pesquería, constituyó la mayor captura con $25.236 \mathrm{~kg}$ ( $\sim 97 \%$ del total en peso) y 109.316 ejemplares (89,4\% del total en número).

En la región de Valparaíso (V) se realizaron 34 lances en 3 caletas (Portales, El Membrillo y San Antonio), principalmente con paños de 52 y $65 \mathrm{~mm}$ de tamaño de malla, registrándose un rendimiento de pesca para todas las especies de 1.910,3 ind. $\mathrm{km}^{-1}$ y 305,1 kg km${ }^{-1}$ (Tabla 1). La participación de merluza común fue $74,4 \%$ del total en número, mientras que la fauna acompañante estuvo compuesta por 15 especies cuyo aporte correspondió al 25,6\% restante (Fig. 2a). Destaca la participación de langostino colorado con el 79,6\% del total de la fauna acompañante en número. De acuerdo a la frecuencia relativa de ocurrencia, langostino colorado y jaiba limón correspondieron a especies comunes dado su registro en más del 50\% de los lances realizados, mientras que langostino amarillo, blanquillo y jaiba paco fueron clasificadas como poco comunes. Las 10 especies restantes fueron clasificadas como raras (Tabla 2).

En la región de O’Higgins (VI) se realizaron 8 lances en una caleta (Bucalemu), usándose en mayor proporción paños de $73 \mathrm{~mm}$ de tamaño de malla. El rendimiento de pesca total correspondió a 1.110,2 ind. $\mathrm{km}^{-1}$ y 304,2 $\mathrm{kg} \mathrm{km}^{-1}$ (Tabla 1). En esta zona se observó que la merluza común aportó el 97,7\% de la captura en número, mientras que otras 5 especies explicaron el 2,3\% restante (Fig. 2b). Al igual que en la región de Valparaíso, langostino colorado fue la especie que más aportó a la fauna acompañante ( $86,5 \%$ del total en número). Tanto langostino colorado como jaiba paco mostraron alta frecuencia de ocurrencia, siendo clasificadas como especies muy comunes en las capturas. Por su parte, 
Tabla 1. Captura total en número (ind) y peso (kg) y captura relativa (en número y peso por km de red) por caleta y tamaño de malla. Se indica también la cantidad de lances realizados y el largo total de las redes / Total catch in number (ind) and weight (kg) and relative catch (in number and weight per $\mathrm{km}$ of net) by fishing cove and mesh size. The number of sets and the total length of the gillnets are also indicated

\begin{tabular}{|c|c|c|c|c|c|c|c|c|}
\hline \multirow[b]{2}{*}{ Caleta } & \multirow{2}{*}{$\begin{array}{l}\text { Tamaño de } \\
\text { malla }(\mathrm{mm})\end{array}$} & \multirow[b]{2}{*}{ Lances } & \multirow{2}{*}{$\begin{array}{l}\text { Profundidad } \\
\text { (m) }\end{array}$} & \multirow{2}{*}{$\begin{array}{c}\text { Largo total } \\
\text { de las redes } \\
(\mathrm{m})\end{array}$} & \multicolumn{4}{|c|}{ Captura } \\
\hline & & & & & Ind & ind. $\mathrm{km}^{-1}$ & $\mathrm{~kg}$ & $\mathrm{~kg} \mathrm{~km}^{-1}$ \\
\hline \multicolumn{9}{|c|}{ Región de Valparaíso (V) } \\
\hline El Membrillo & 52 & 13 & $60-120$ & $5.970,5$ & 9.004 & $1.508,1$ & $1.489,7$ & 249,5 \\
\hline Portales & 52 & 9 & $90-120$ & $8.190,0$ & 26.444 & $3.228,8$ & $3.310,3$ & 404,2 \\
\hline San Antonio & 65 & 10 & $70-150$ & $8.820,0$ & 9.659 & $1.095,1$ & $2.321,0$ & 263,2 \\
\hline San Antonio & 73 & 2 & $120-180$ & $1.190,0$ & 1.065 & 895,0 & 252,8 & 212,4 \\
\hline Sub-total & & 34 & & $24.170,5$ & 46.172 & $1.910,3$ & $7.373,8$ & 305,1 \\
\hline \multicolumn{9}{|c|}{ Región de O’Higgins (VI) } \\
\hline Bucalemu & 65 & 2 & $130-140$ & $2.240,0$ & 2.360 & $1.053,6$ & 640,6 & 286,0 \\
\hline Bucalemu & 73 & 6 & $110-140$ & $6.177,6$ & 6.985 & $1.130,7$ & $1.919,8$ & 310,8 \\
\hline Sub-total & & 8 & & $8.417,6$ & 9.345 & $1.110,2$ & $2.560,4$ & 304,2 \\
\hline \multicolumn{9}{|c|}{ Región del Maule (VII) } \\
\hline Curanipe & 65 & 5 & $30-50$ & $6.256,3$ & 13.976 & $2.233,9$ & $3.105,6$ & 496,4 \\
\hline Duao & 65 & 9 & $30-60$ & $7.048,4$ & 7.764 & $1.101,5$ & $1.766,4$ & 250,6 \\
\hline Maguillines & 65 & 7 & $20-40$ & $5.200,0$ & 10.023 & $1.927,5$ & $2.331,3$ & 448,3 \\
\hline Sub-total & & 21 & & $18.504,7$ & 31.763 & $1.716,5$ & $7.203,3$ & 389,3 \\
\hline \multicolumn{9}{|c|}{ Región del Biobío (VIII) } \\
\hline Cocholgüe & 73 & 17 & $95-220$ & $9.585,9$ & 14.700 & $1.533,5$ & $3.646,9$ & 380,4 \\
\hline Coliumo & 65 & 3 & $70-90$ & $2.826,7$ & 5.246 & $1.855,9$ & $1.545,7$ & 546,8 \\
\hline Laraquete & 73 & 2 & $40-95$ & $2.300,0$ & 1.449 & 630,0 & 397,1 & 172,7 \\
\hline Lo Rojas & 85 & 3 & $75-95$ & $3.600,0$ & 1.027 & 285,3 & 324,4 & 90,1 \\
\hline Quidico & 85 & 2 & $110-160$ & $2.400,0$ & 1.178 & 490,8 & 268,3 & 111,8 \\
\hline Tubul & 73 & 2 & $80-105$ & $3.024,0$ & 2.729 & 902,4 & 640,4 & 211,8 \\
\hline Tumbes & 85 & 6 & $70-140$ & $9.000,0$ & 8.727 & 969,7 & $2.048,0$ & 227,6 \\
\hline Sub-total & & 35 & & $32.736,5$ & 35.056 & $1.070,9$ & $8.870,8$ & 271,0 \\
\hline Total & & 98 & & $83.829,3$ & 122.336 & $1.459,3$ & $26.008,3$ & 310,3 \\
\hline
\end{tabular}

lenguado y jaiba limón correspondieron a especies poco comunes (Tabla 2).

En la región del Maule (VII) se realizaron 21 lances en 3 caletas (Curanipe, Duao y Maguillines) utilizándose exclusivamente paños de $65 \mathrm{~mm}$ de tamaño de malla. El rendimiento de pesca correspondió a 1.716,5 ind. $\mathrm{km}^{-1} \mathrm{y}$ $389,3 \mathrm{~kg} \mathrm{~km}^{-1}$ (Tabla 1). En esta zona se registró el mayor número de especies capturadas (18 especies en total), no obstante merluza común aportó el 98,5\% del total en número (Fig. 2c). La captura de jaiba limón correspondió al $41,3 \%$ de la fauna acompañante seguida de lenguado $(18,3 \%)$ y jaiba paco (12,4\%). Según la frecuencia relativa de ocurrencia, sólo jaiba limón fue clasificada como especie muy común, mientras que lenguado correspondió a una especie común (Tabla 2). Por su parte, jaiba paco, blanquillo y pampanito fueron clasificadas como especies poco comunes, y las restantes 11 especies fueron especies raras.

En la región del Biobío (VIII) se realizaron 35 lances en 7 caletas (Cocholgüe, Coliumo, Laraquete, Lo Rojas, Quidico, Tubul y Tumbes) usándose principalmente paños de 73 y $85 \mathrm{~mm}$ de tamaño de malla. El rendimiento de pesca fue de 1.070,9 ind. $\mathrm{km}^{-1}$ y $271 \mathrm{~kg} \mathrm{~km}^{-1}$ (Tabla 1). El aporte de merluza común en esta zona fue del $98,6 \%$ en número, mientras que las restantes 9 especies sólo aportaron un 1,4\% (Fig. 2d). Las principales especies que componen la fauna acompañante fueron langostino colorado y lenguado las que aportaron el 51 y 36\% del 
Tabla 2. Número de ejemplares capturados por especie y región. Entre paréntesis, la clasificación de cada especie según: R= rara; $\mathrm{U}=$ poco común; $\mathbf{C}=$ común; $\mathbf{V}=$ muy común. $\mathbf{N}=$ número de lances totales por región. *Especie objetivo / Number of individuals caught by species and region. In parenthesis, the classification of each species according to: $\mathrm{R}=\mathrm{rare}$ species; $\mathrm{U}=\mathrm{uncommon}$ species; $\mathrm{C}=$ common species; $\mathrm{V}=$ very common species. $\mathrm{N}=$ number of total sets performed by region. *Target species

\begin{tabular}{|c|c|c|c|c|}
\hline \multirow{2}{*}{ Taxa } & \multicolumn{4}{|c|}{ Región } \\
\hline & $\mathrm{V}(\mathrm{N}=34)$ & VI $(\mathrm{N}=8)$ & VII $(\mathrm{N}=21)$ & VIII $(\mathrm{N}=35)$ \\
\hline \multicolumn{5}{|l|}{ Vertebrados } \\
\hline \multicolumn{5}{|l|}{ Mixines } \\
\hline Anguila Eptatretus polytrema (Girard, 1855) & $1(\mathrm{R})$ & & & \\
\hline \multicolumn{5}{|l|}{ Condrictios } \\
\hline Pintarroja Schroederichthys chilensis (Guichenot, 1848) & $5(\mathrm{R})$ & & $13(\mathrm{R})$ & $2(\mathrm{R})$ \\
\hline Pejegallo Callorhinchus callorynchus (Linnaeus, 1758) & & & $2(\mathrm{R})$ & \\
\hline \multicolumn{5}{|l|}{ Osteíctios } \\
\hline Blanquillo Prolatilus jugularis (Valenciennes, 1833) & $243(U)$ & & $36(U)$ & $8(\mathrm{R})$ \\
\hline Cabrilla Sebastes oculatus Valenciennes, 1833 & & & & $4(\mathrm{R})$ \\
\hline Congrio colorado Genypterus chilensis (Guichenot, 1848) & $1(\mathrm{R})$ & & & \\
\hline Congrio negro Genypterus maculatus (Tschudi, 1846) & $4(\mathrm{R})$ & $1(\mathrm{R})$ & $16(U)$ & $7(\mathrm{R})$ \\
\hline Corvina Cilus gilberti (Abbott, 1899) & $1(\mathrm{R})$ & & $1(\mathrm{R})$ & \\
\hline Jurel Trachurus murphyi Nichols, 1920 & $9(\mathrm{R})$ & & $3(\mathrm{R})$ & \\
\hline Lenguado Hippoglossina macrops Steindachner, 1876 & $18(\mathrm{R})$ & $3(U)$ & $90(\mathrm{C})$ & $173(\mathrm{C})$ \\
\hline Merluza común* Merluccius gayi gayi (Guichenot, 1848) & $34.339(\mathrm{~V})$ & $9.130(\mathrm{~V})$ & $31.271(\mathrm{~V})$ & $34.576(\mathrm{~V})$ \\
\hline Pampanito Stromateus stellatus Cuvier, 1829 & & & $33(\mathrm{U})$ & \\
\hline Sierra Thyrsites atun (Euphrasen, 1791) & & & $8(\mathrm{R})$ & $2(\mathrm{R})$ \\
\hline \multicolumn{5}{|l|}{ Invertebrados } \\
\hline \multicolumn{5}{|l|}{ Cnidarios } \\
\hline Coral Clase Anthozoa & & & $6(\mathrm{R})$ & \\
\hline \multicolumn{5}{|l|}{ Moluscos } \\
\hline Pulpo Octopus sp. & & & $9(\mathrm{R})$ & \\
\hline Jibia Dosidicus gigas (d'Orbigny, 1835) & $9(\mathrm{R})$ & & & \\
\hline Caracol Aeneator sp. & & & $3(\mathrm{R})$ & \\
\hline \multicolumn{5}{|l|}{ Crustáceos } \\
\hline Jaiba limón Cancer porteri Rathbun, 1930 & $1.090(\mathrm{C})$ & $3(\mathrm{U})$ & $203(V)$ & $7(\mathrm{R})$ \\
\hline Jaiba mora Homalaspis plana (Milne Edwards, 1834) & $1(\mathrm{R})$ & & & \\
\hline Jaiba paco Platymera gaudichaudii Milne Edwards, 1837 & $86(U)$ & $22(\mathrm{~V})$ & $61(\mathrm{U})$ & $33(\mathrm{R})$ \\
\hline Langostino amarillo Cervimunida johni Porter, 1903 & $942(\mathrm{U})$ & & & \\
\hline Langostino colorado Pleuroncodes monodon (Milne Edwards, 1837) & $9.422(\mathrm{C})$ & $186(V)$ & & $244(\mathrm{C})$ \\
\hline \multicolumn{5}{|l|}{ Equinodermos } \\
\hline Erizo negro Tetrapygus niger (Molina, 1782) & $1(\mathrm{R})$ & & $6(\mathrm{R})$ & \\
\hline Estrella Clase Asteroidea & & & $1(\mathrm{R})$ & \\
\hline \multicolumn{5}{|l|}{ Ascidia } \\
\hline Piure Pyura chilensis Molina, 1782 & & & $1(\mathrm{R})$ & \\
\hline
\end{tabular}

total en número, respectivamente; siendo ambas clasificadas como especie comunes de acuerdo a su frecuencia relativa de ocurrencia (Tabla 2). Las 7 especies restantes fueron clasificadas como raras.

El análisis de agrupamiento de la captura estandarizada y transformada permitió identificar 4 grupos principales de lances (grupos 1 al 4; Fig. 3a) a un nivel de similitud del $60 \%$ o superior. Un quinto grupo, conformado sólo por 5 lances, no fue considerado por su baja representatividad. Por su parte, el análisis de ordenación mostró los mismos 4 grupos de lances identificados en el análisis de agrupamiento, con un nivel de estrés aceptable (estrés=0,13; Fig. 3b), validando el criterio de similitud del dendrograma y la identificación de 4 ensambles comunitarios. Estos grupos responden tanto a patrones latitudinales como batimétricos. 


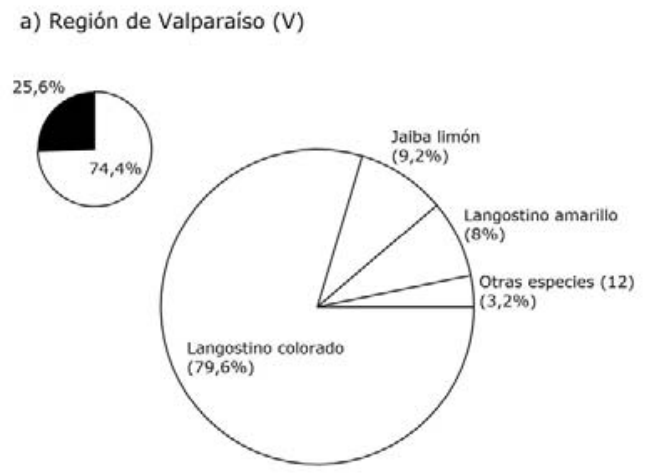

b) Región de O'Higgins (VI)

c) Región del Maule (VII)

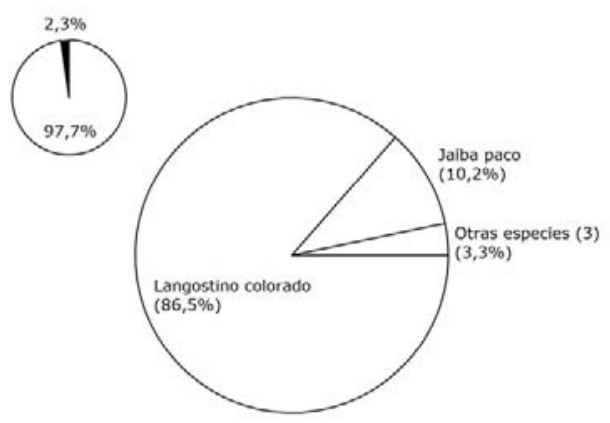

d) Región del Biobio (VIII)
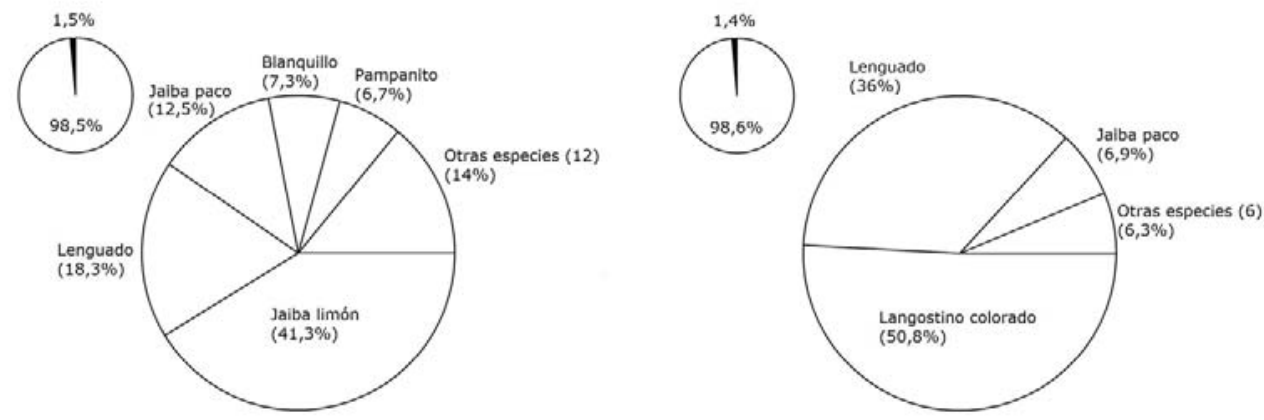

Figura 2. Composición de especies de la captura (en número) con redes de enmalle por región. Los gráficos pequeños muestran la proporción de merluza común (en blanco) y de otras especies (en negro). Los gráficos mayores muestran las especies que componen la fauna acompañante / Species composition of the catch (in number) per region using gillnets. The smaller pies show the proportion of Chilean hake (in white) and the others species (in black). The bigger pies show the species that compose the bycatch

En particular, el Grupo 1 se conformó por lances realizados en las regiones $\mathrm{V}$ y VII en el estrato más somero $(<50 \mathrm{~m})$ usando redes con mallas de 52 y $65 \mathrm{~mm}$. En este grupo, merluza común y jaiba limón fueron las especies que más aportaron a explicar la similitud (67,7 y 23,9\%, respectivamente). Por su parte, el Grupo 2 lo conformaron lances realizados entre la $\mathrm{V}$ y VIII regiones en los estratos intermedio y profundo (>100 m), empleando redes con mallas entre 65 y $86 \mathrm{~mm}$. La similitud del grupo estuvo determinada por la contribución de merluza común $(82,4 \%)$ y langostino colorado (13,9\%). El Grupo 3 se conformó por lances realizados en la zona sur (VII y VIII regiones) en los estratos somero e intermedio ( $<150 \mathrm{~m}$ ), usando redes con mallas entre 65 y $86 \mathrm{~mm}$. En este grupo la similitud fue explicada por merluza común (71,5\%), lenguado $(17,2 \%)$ y blanquillo (4,6\%). Por último, el Grupo 4 se constituyó por lances realizados exclusivamente en la $\mathrm{V}$ Región en el estrato intermedio (50-150 m), usando redes con mallas pequeñas (52 mm). La similitud de este grupo estuvo explicada por la participación de merluza común (45,6\%), langostino colorado (27,1\%) y jaiba limón (18,1\%).

\section{Discusión}

Si bien las redes de enmalle se caracterizan por tener una baja selectividad de captura de especie (Suuronen et al. 2012), los resultados obtenidos en este trabajo indicaron que la pesquería artesanal de merluza común con redes de enmalle vulneran un bajo número de especies, siendo además un menor aporte relativo de éstas a la captura total en calidad de fauna acompañante. En este sentido, se identificó la presencia de 24 taxones junto a la especie objetivo, donde la mayor cantidad (16 especies) se observaron en menos del $25 \%$ de los lances realizados, clasificándose en este caso como especies raras. Sólo 8 especies presentaron en una o más regiones, una frecuencia relativa de ocurrencia mayor o igual al 25\% de los lances, donde 4 de ellas se destacan por su aporte relativo al total de la fauna acompañante. Estas especies correspondieron a 3 crustáceos (jaiba limón, jaiba paco y langostino colorado) y un pez óseo (lenguado), todas de hábitos bentodemersales. 

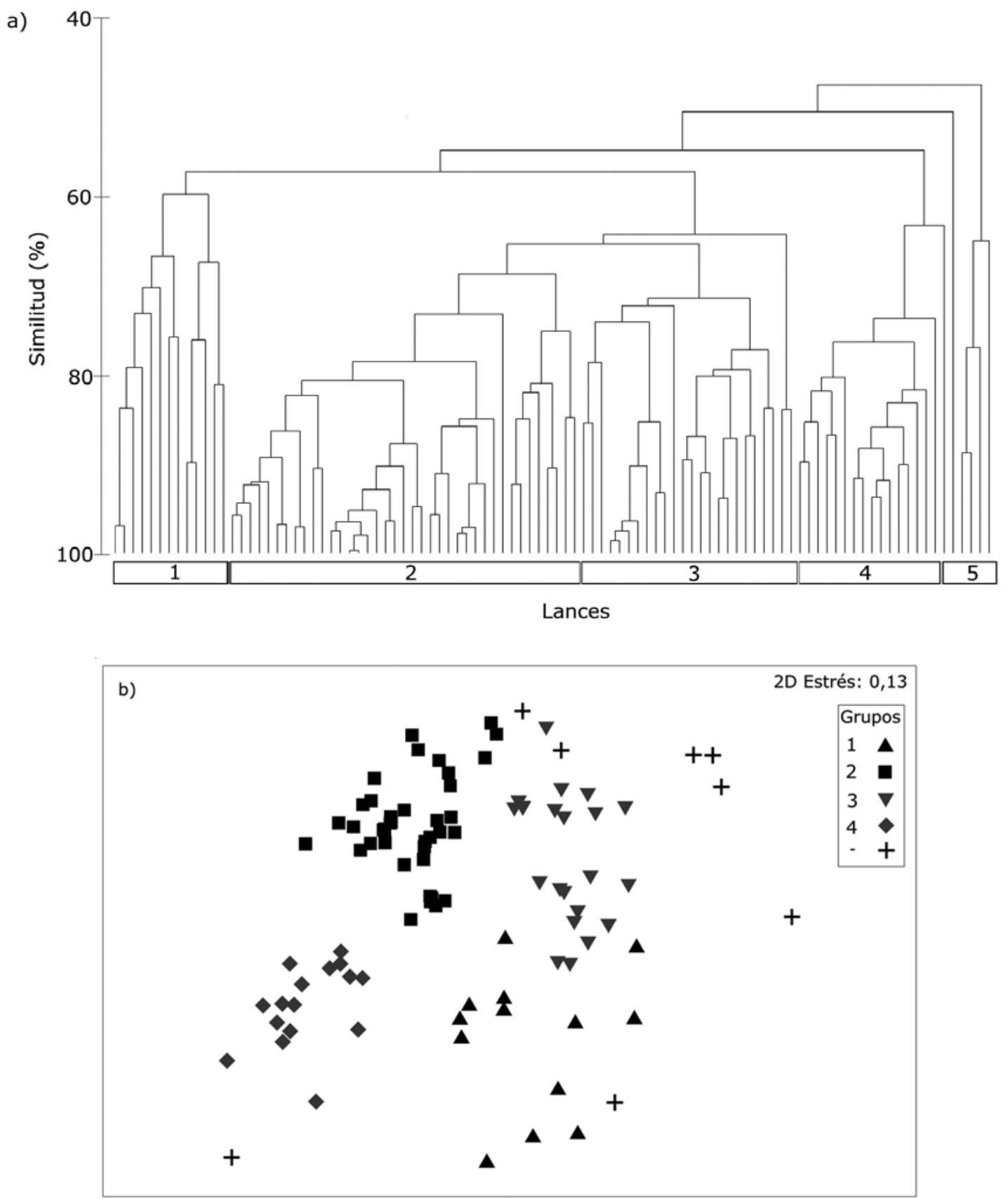

Figura 3. Dendrograma para el agrupamiento de los lances (a) y ordenación MDS (b) basado en la similitud de Bray-Curtis para la captura en número (datos estandarizados y transformados) obtenida en todos los lances / Dendrogram for groupaverage clustering (a) and MDS ordination (b) based on Bray-Curtis similarities for catch in numbers (standardized and transformed data) obtained from all sets 
No existen antecedentes comparables respecto a la composición de especies capturadas con redes de enmalle en el área de estudio, no obstante se han reportado algunos resultados con redes de arrastre referidos a asociaciones faunísticas. En este sentido, Acuña et al. (2005) establecen que merluza común, langostino amarillo, langostino colorado y lenguado conforman un ensamble somero (entre 50 y 199 m), mientras que Melo et al. (2007) indican que merluza común, jaiba paco y langostino amarillo son especies típicas entre 100 y 200 m de profundidad, destacando que este patrón es variable entre zonas (entre $33^{\circ}$ y 38³0’S). Arancibia (1992) también describe cambios espaciales en las asociaciones de peces principalmente en términos de la profundidad, sin considerar la dimensión horizontal (latitudinal) por limitación de información.

Ciertamente, la composición de los ensambles es variable en términos espaciales y temporales, y responde tanto a aspectos tróficos entre las especies más abundantes (Arancibia 1991) como a las características del hábitat. Estas diferencias han sido asociadas a las condiciones abióticas de temperatura, salinidad y oxígeno disuelto, tanto por la dinámica propia del sistema de corriente de Humboldt (Thiel et al. 2007) como por la variabilidad en la intensidad y profundidad de la zona de mínimo oxígeno (Atkinson et al. 2002).

A partir de los resultados obtenidos, las condiciones ambientales podrían explicar parcialmente las diferencias en las especies capturadas, su participación relativa y el agrupamiento obtenido entre diferentes zonas de pesca, esto reflejado en que los 4 grupos resultantes del análisis están asociados a un patrón latitudinal y batimétrico diferenciado. Si bien el tamaño de malla también puede jugar un rol importante en la selección de especies, los resultados obtenidos indicarían que esta variable es secundaria, probablemente afectando en mayor grado la cantidad de ejemplares de una especie que son capturados por la red (Queirolo et al. 2013b) más que la diversidad de especies en sí misma.

Como ha sido tradicional en Chile central, la pesquería artesanal de enmalle de merluza común tiene un carácter mono-específico (Gálvez et al. 2013), por lo tanto la mayor parte de la fauna acompañante conforma el descarte realizado por la flota, salvo excepciones como el congrio negro, congrio colorado y corvina, según el tamaño de los ejemplares. Las restantes especies son, por lo general, devueltas al mar ya sean vivas o muertas. Aunque se reconoce que el descarte constituye una práctica no deseada (Kelleher 2005), el hecho de que la mayoría de las especies no tenga valor comercial constituye la principal causa para su devolución. Si bien los volúmenes de descarte serían bajos de acuerdo a la participación relativa y la frecuencia de ocurrencia registrada a bordo, uno de los mayores inconvenientes corresponde a la captura de crustáceos, particularmente langostinos y jaibas. Estas especies se enredan y dificultan su remoción durante la limpieza de la red, hecho que motiva la necesidad de evaluar mejoras tecnológicas en el diseño del enmalle para evitar el contacto del paño con el fondo marino y así reducir tanto la captura de especies no objetivo como interferencias operacionales indeseadas.

\section{Agradecimientos}

Este estudio fue parcialmente financiado por el Fondo de Investigación Pesquera mediante el Proyecto FIP N²00923 'Caracterización de las redes de enmalle en la pesquería artesanal de merluza común’. Los autores agradecen las facilidades brindadas por los armadores, patrones y tripulaciones durante las salidas de pesca en todas las caletas. Del mismo modo, agradecemos a Gonzalo Valenzuela, David Valdés y Francisco Gallardo por su colaboración durante los muestreos. Los autores agradecen a los tres evaluadores quienes realizaron valiosas sugerencias y aportes que contribuyeron a mejorar este trabajo.

\section{LITERATURA CITADA}

Acuña E, JC Villaroel, M Andrade \& A Cortés. 2005. Fauna acompañante en pesquerías de arrastre de crustáceos de Chile: implicancias y desafíos desde la perspectiva de la biodiversidad. En: Figueroa E (ed). Biodiversidad marina: valoración, usos y perspectivas ¿hacia dónde va Chile?, pp. 395-424. Editorial Universitaria, Santiago.

Aguayo M. 1995. Biology and fisheries of Chilean hakes ( $M$. gayi and $M$. australis). In: Alheit J \& TJ Pitcher (eds). Hakes: Biology, fisheries and markets, pp. 305-338. Chapman \& Hall, Great Britain.

Arancibia H. 1991. Análisis ecológico-pesquero del recurso langostino colorado (Pleuroncodes monodon) y su interacción con merluza común (Merluccius gayi) y lenguado de ojos grandes (Hippoglossina macrops). Biología Pesquera 20: 37-48.

Arancibia H. 1992. Distribution patterns of the demersal fish assemblage off Central Chile. Biología Pesquera 21: 43-53.

Atkinson LP, A Valle-Levinson, D Figueroa, R de Pol-Holz, VA Gallardo, W Schneider, JL Blanco \& M Schmidt. 2002. Oceanographic observations in Chilean coastal waters between Valdivia and Concepción. Journal of Geophysical Research 107: 1-13. 
Clarke KR \& RM Warwick 2001. Change in marine communities: an approach to statistical analysis and interpretation, 172 pp. PRIMER-E, Plymouth.

Field JG, KR Clarke \& RM Warwick. 1982. A practical strategy for analysis of multispecies distribution patterns. Marine Ecology Progress Series 8: 37-52.

Gálvez P, J Sateler, A Flores, Z Young, J Olivares, K Riquelme \& J González. 2013. Pesquerías demersales y aguas profundas, 2012. Sección II: Demersales centro sur, 2012, 162 pp. Seguimiento General de Pesquerías de Peces y Crustáceos, SUBPESCA / IFOP, Valparaíso.

Kelleher K. 2005. Discarding in the world's marine fisheries: an update. FAO Fisheries Technical Paper 470: 1-131.

Kong I \& R Meléndez. 1991. Estudio taxonómico y sistemático de la ictiofauna de aguas profundas capturadas entre Arica e Isla Mocha (18³0'-30³0’ S). Estudios Oceanológicos 10: 1-81.

Meléndez R \& D Meneses. 1989. Tiburones del talud continental recolectados entre Arica (18²'S) e Isla Mocha (38³0’S), Chile. Investigaciones Marinas 17: 3-73.

Melo T, N Silva, P Muñoz, J Díaz-Naveas, J Sellanes, A Bravo, J Lamilla, J Sepúlveda, R Vögler, Y Guerrero, C Bustamante, MA Alarcón, D Queirolo, F Hurtado, E Gaete, P Rojas, I Montenegro, R Escobar \& V Zamora. 2007. Caracterización del fondo marino entre la III y X Regiones. Informe Final FIP-IT/2005-61: 1-287. <http:// www.fip.cl/Archivos/Hitos/Informes/inffinal\%20200561.pdf $>$

Pequeño G. 1997. Peces de Chile. Lista sistemática revisada y comentada. Revista de Biología Marina 24: 1-132.

Queirolo D, E Gaete, M Ahumada, T Melo, J Merino \& R Escobar. 2011. Caracterización de las redes de enmalle en la pesquería artesanal de merluza común. Informe Final FIPIT /2009-23: 1-117. <http://www.fip.cl / Archivos / Hitos /Informes/INFORME \% 20HITO \% 2 0FINAL168Adjunto 1.pdf $>$

Queirolo D, E Gaete \& M Ahumada. 2013a. Gillnet selectivity for Chilean hake (Merluccius gayi gayi Guichenot, 1848) in the bay of Valparaíso. Journal of Applied Ichthyology 29: 775-781.
Queirolo D, E Gaete, M Ahumada, F Hurtado, J Merino, I Montenegro, R Escobar \& V Zamora. 2013b. Selectividad de redes de enmalle en la pesquería artesanal de merluza común. Informe Final FIP-IT/2011-10: 1-71. http:// www.fip.cl/Archivos/Hitos/Informes/I N F O R M E \% 20 HITO\%20FINAL1043Adjunto1.pdf

Retamal MA. 1981. Catálogo ilustrado de los crustáceos decápodos de Chile. Gayana Zoología 44: 1-110.

Santos M, M Gaspar, C Monteiro \& P Vasconcelos. 2002. Gill net and long-line catch comparisons in a hake fishery: the case of southern Portugal. Scientia Marina 66(4): 433441.

Sparre P \& SC Venema. 1997. Introducción a la evaluación de recursos pesqueros tropicales. Parte 1. Manual. FAO Documento Técnico de Pesca 306(1), Rev. 2: 1-420.

Stergiou KI, DK Moutopoulos \& K Erzini. 2002. Gill net and longlines fisheries in Cyclades waters (Aegean Sea): species composition and gear competition. Fisheries Research 57: 25-38.

Stergiou KI, DK Moutopoulos, MC Soriguer, E Puente, PG Lino, C Zabala, P Monteiro, LA Errazkin \& K Erzini. 2006. Trammel net catch species composition, catch rates and métiers in southern European waters: A multivariate approach. Fisheries Research 79: 170-182.

Suuronen P, F Chopin, C Glass, S Løkkeborg, Y Matsushita, D Queirolo \& D Rihan. 2012. Low impact and fuel efficient fishing-Looking beyond the horizon. Fisheries Research 119-120: 135-146.

Thiel M, EC Macaya, E Acuña, WE Arntz, H Bastias, K Brokordt, PA Camus, JC Castilla, LR Castro, M Cortés, CP Dumont, R Escribano, M Fernandez, JA Gajardo, CF Gaymer, I Gómez, AE González, HE González, PA Haye, JE Illanes, JL Iriarte, DA Lancellotti, G LunaJorquera, C Luxoro, PH Manríquez, V Marín, P Muñoz, SA Navarrete, E Perez, E Poulin, J Sellanes, HH Sepúlveda, W Stotz, F Tala, A Thomas, CA Vargas, JA Vásquez \& JMA Vega. 2007. The Humboldt Current System of northern and central Chile-Oceanographic processes, ecological interactions and socioeconomic feedback. Oceanography and Marine Biology: An Annual Review 45: 195-344. 